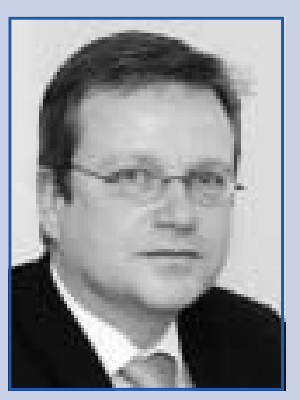

Prof. Dr. Jochen

Zimmermann ist Professor an der Universität Bremen und Inhaber des Lehrstuhls für Allgemeine Betriebswirtschaftslehre, Unterneh mensrechnung und Controlling. E-Mail: jzimmermann@unibremen.de.

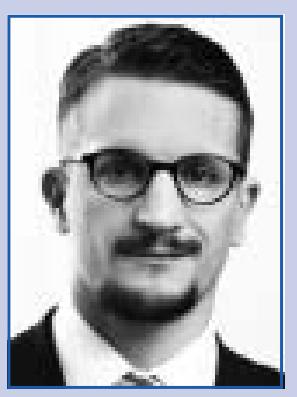

Dr. Jan-Philipp Kilian ist nach seiner Promotion an der Universität Bremen bei der PricewaterhouseCoopers AG in Frankfurt/Main im Bereich Financial Services Consulting tätig. E-Mail: jan-philipp. kilian@de.pwc.de.

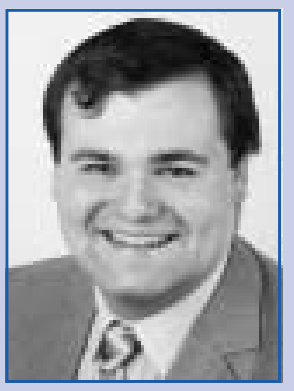

Sebastian A. Tideman promoviert an der Universität Bremen und arbeitet als wissenschaftlicher Mitarbeiter am Lehrstuhl für Allgemeine Betriebswirtschaftslehre, Unterneh mensrechnung und Controlling. E-Mail: tideman@unibremen.de.

\author{
Stichwörter: \\ - Bilanzierungskonzepte \\ - Covenants \\ - Covenants-Controlling \\ - Gläubigerschutz \\ - Informatorische Bilanzierung
}

\title{
Bilanzierungskonzepte und die Rolle von Covenants
}

\author{
Jochen Zimmermann, Jan-Philipp Kilian und Sebastian A. Tideman
}

Die länderspezifisch unterschiedliche Bedeutung von Covenants bei der Kreditvergabe lässt sich durch verschiedene Bilanzierungskonzeptionen erklären. In Deutschland ist in den letzten Jahren eine zunehmende Verwendung von Covenants zu beobachten. Dieser Beitrag diskutiert Gründe für diese Entwicklung und leitet Folgerungen für das Controlling der Kreditnehmer ab.

\section{Gläubigerschutz im Wandel}

Vergleicht man die Verbreitung von Covenants, so fällt ihre länderspezifisch unterschiedliche Bedeutung für Kreditvergabeentscheidungen ins Auge (vgl. Velte, 2007). Dieser Beitrag untersucht diese ungleiche Verbreitung der Covenants und zeigt, dass Bilanzierungskonzepte die in Abb. 1 gezeigten Orientierungen am Gläubigerschutz einerseits und an einer informatorischen Rechnungslegung andererseits - einen wesentlichen Einfluss auf die Verwendungshäufigkeit von Covenants haben.

Die beiden Bilanzierungskonzepte und die damit einhergehenden Rollen der Covenants werden als Ausgangspunkt für die Diskussion dienen, welche Implikationen sich für das Controlling in den jeweiligen Rechnungslegungssystemen ergeben. Diese Analyse erfolgt beispielhaft anhand der Länderfälle USA und Deutschland, die als Repräsentanten einer informatorischen Rechnungslegung (USA) respektive gläubigerschutzorientierten Rechnungslegung (Deutschland) gelten (vgl. Zimmermann et al., 2012).

Covenants, also nebenvertragliche Kreditvereinbarungen, sind zunächst aus dem angelsächsischen Raum bekannt; aber auch in Deutschland ist eine zunehmende Verbreitung der Covenants zu beobachten. So verwendeten schon im Jahr 2009 gemäß einer Studie von Bantleon et al. (2009) nahezu drei Viertel der untersuchten Banken zumindest gelegentlich Covenants - mit zunehmender Tendenz. Hierdurch entstehen Konsequenzen für das Controlling der Kreditneh- mer, auf die im Folgenden einzugehen sein wird.

In bisherigen Veröffentlichungen wurde zumeist die zunehmende Verbreitung der Covenants in Deutschland beleuchtet. Es mangelt aber an einer Analyse, welche Konsequenzen sich hieraus für das $\mathrm{Zu}$ sammenspiel zwischen Controlling und externem Rechnungswesen sowie für die Struktur des Controllings ergeben. Diese für die Unternehmenspraxis relevante Fragestellung behandelt dieser Beitrag. Er ist wie folgt aufgebaut: In Abschnitt 2 werden zunächst die unterschiedlichen Bilanzierungskonzepte vorgestellt. Abschnitt 3 thematisiert sodann die Verbreitung der Covenants in einer länderübergreifenden Analyse, woraufhin in Abschnitt 4 Implikationen für das Controlling abgeleitet werden. Abschnitt 5 fasst diesen Beitrag zusammen.

\section{Bilanzierungskonzepte}

Die Bedeutung von funktionierenden Kapitalmärkten für die Wohlfahrt eines Landes ist unbestritten. Rechnungslegungsregeln eines Landes nehmen für die Arbeitsweise von Kapitalmärkten eine wichtige Rolle ein (vgl. Meser et al., im Erscheinen 2015). Ihre kapitalmarktorientierte Gestaltung zielt dabei vor allem auf die Bereitstellung von entscheidungsnützlichen Informationen für Investoren ab. Insbesondere die United States Generally Accepted Accounting Principles (USGAAP) sowie die International Financial Reporting Standards (IFRS) sind prominente Beispiele für eine informatorische Rechnungslegung (vgl. Kahle, 2002). Im 


\begin{tabular}{|l|l|l|}
\hline & $\begin{array}{l}\text { Gläubigerschutzorientierte } \\
\text { Rechnungslegung }\end{array}$ & $\begin{array}{l}\text { Informatorische } \\
\text { Rechnungslegung }\end{array}$ \\
\hline Ziel & Kapitalerhaltung & $\begin{array}{l}\text { Bereitstellung entscheidungs- } \\
\text { nützlicher Informationen }\end{array}$ \\
\hline $\begin{array}{l}\text { Zugehörige Bilanz- } \\
\text { auffassung }\end{array}$ & $\begin{array}{l}\text { Revenue Expense Approach/ } \\
\text { dynamische Bilanztheorie }\end{array}$ & $\begin{array}{l}\text { Asset Liability Approach/ } \\
\text { statische Bilanztheorie }\end{array}$ \\
\hline Primäres Rechenwerk & $\begin{array}{l}\text { GuV mit Bilanz als } \\
\text { Nebenprodukt }\end{array}$ & $\begin{array}{l}\text { Bilanz mit GuV als } \\
\text { Nebenprodukt }\end{array}$ \\
\hline $\begin{array}{l}\text { Bevorzugte Vermögens- } \\
\text { bewertungsmethode }\end{array}$ & Fortgeführte Anschaffungskosten & Fair Value \\
\hline Leitmotiv & Verlässlichkeit & Relevanz \\
\hline Zeitperspektive & Eher vergangenheitsorientiert & Eher zukunftsorientiert \\
\hline
\end{tabular}

Abb. 1: Bilanzierungskonzepte im Vergleich

Gegensatz dazu gibt es Regeln, die die Bereitstellung von entscheidungsnützlichen Informationen höchstens als Nebenziel formulieren und vielmehr die Anspruchsbemessung und den Gläubigerschutz als wesentliches Ziel verfolgen, wie beispielsweise die Rechnungslegungsvorschriften des deutschen Handelsgesetzbuchs (HGB). Bezogen auf die Bilanzierung ergibt sich dadurch ein gegenläufiges Konzept: Informatorische Rechnungslegungsvorschriften versuchen relevante Informationen bereit $\mathrm{zu}$ stellen und schreiben deshalb häufig eine vollständige, zeitnahe und an Marktwerten orientierte Bilanzierung der Vermögenswerte und Schulden vor, um eine möglichst hohe Angleichung der Bewertungsergebnisse des Rechnungswesens an eine Marktbewertung zu sichern. Das Problem bei dieser als Relevanzsicherung bezeichneten Vorgehensweise ist jedoch, dass mit einer Zunahme einer so verstandenen Relevanz der Bewertung ebenfalls eine Zunahme der Schwankungen sowie eine Abnahme der Verlässlichkeit einhergehen.

Das HGB hingegen verankert in $\$ 252$ Abs. 1 das Vorsichtsprinzip. Hierdurch ergeben sich häufig niedrige und wegen des Anschaffungskostenprinzips weniger zeitnahe Bewertungsansätze der Vermögenswerte und hohe Bewertungsansätze der Schulden, insbesondere Rückstellungen betreffend. Dieses Vorgehen sorgt für eine größere Verlässlichkeit des Jahresabschlusses, zumindest in Form geringerer Schwankungen der Ergebnisse und Wertansätze, und verringert ebenso die Wahrscheinlichkeit, dass das Unternehmen überhöhte Dividenden ausschüttet und damit die Zugriffsmasse für die Gläubiger im Krisenfall verringert.
Diese unterschiedlichen Ansätze haben Auswirkungen auf Kreditbeziehungen. Gläubiger sind dem Risiko ausgesetzt, dass der Darlehensnehmer seinen Kredit nicht zurückzahlen kann. Damit Fremdkapitalgeber $\mathrm{zu}$ dessen Kompensation nicht prohibitiv hohe Risikoprämien verlangen, haben die kreditsuchenden Unternehmen selbst ein Interesse daran, dass die Gläubigerinteressen angemessen berücksichtigt werden. In gläubigerschutzorientierten Ländern wie Deutschland ist in Form von Ausschüttungssperren sowie einer am Vorsichtsprinzip orientierten Bilanzierung bereits für einen starken Gläubigerschutz gesorgt. In den USA oder anderen Ländern mit informatorischer Rechnungslegung existieren jedoch kaum solche standardisierten Vorschriften, die die Gläubigerposition stärken. Somit ergibt sich ein Handlungsbedarf, den Gläubigerschutz auf individueller und einzelvertraglicher Ebene zu gewährleisten. Dies kann durch den Einsatz von Covenants geschehen. Neben der Einhaltung von finanziellen Covenants bieten auch informative Covenants den Gläubigern einen besseren Schutz, indem sie die Kontrolle des Kreditnehmers erleichtern. Vor dem Hintergrund, dass die Manipulation von Finanzdaten eine der häufigsten wirtschaftskriminellen Straftaten weltweit ist, erscheint der Abbau der Informationsasymmetrien und eine stärkere Überwachung der Kreditnehmer rational (vgl. KPMG, 2011).

\section{Covenants im länderübergrei- fenden Vergleich}

Während Covenants in den USA seit jeher eine wichtige Rolle einnehmen, zeigt sich in Europa ein anderes Bild: So finden
Covenants erst seit einigen Jahren verstärkt Verwendung (vgl. Smith/Warner, 1979; Zimmermann et al., 2012). Lediglich Großbritannien gilt innerhalb Europas als ein Land, in dem Covenants bei der Kreditvergabe seit längerem regelmäßig vereinbart werden (vgl. Omrod/Taylor, 2004). Was die Verbreitung der Covenants in Deutschland betrifft, ergibt sich ein differenziertes Bild: Während die Verwendung von Covenants bei kleineren und mittleren Banken nach wie vor eher ungebräuchlich ist, ist sie bei Großbanken zunehmend üblich (vgl. Zimmermann et al. 2011). Dabei werden Covenants nicht nur vermehrt bei Vertragsabschluss in den Kreditvertrag integriert, sondern ebenfalls als Gegenmaßnahme bei einer Verschlechterung der Kreditnehmerbonität ergriffen.

Die zunehmende Verbreitung der Covenants in Deutschland ist auf verschiedene Faktoren zurückzuführen. Insbesondere das Bilanzrechtsmodernisierungsgesetz (BilMoG) aus dem Jahr 2009 sorgte für eine graduelle Abkehr vom Vorsichtsprinzip und zur Verringerung eines rechnungslegungsbasierten Gläubigerschutzes. Die Regeln des BilMoG haben nicht nur eine Auswirkung auf Gewinnund Verlustrechnungs (GuV)-Kennzahlen, sondern sie beeinflussen ebenso Bilanzkennzahlen. Ein Beispiel soll an dieser Stelle das Zusammenspiel zwischen Bilanz und $\mathrm{GuV}$ verdeutlichen: Erfolgt die Aktivierung eines selbsterstellten immateriellen Vermögenswertes in der Entwicklungsphase, hat dies nicht nur durch die Aktivierung des Aufwandes einen höheren Gewinn zur Folge. Es vergrößern sich auch die Bilanzsumme und Eigenkapitalquote, da durch die Aktivierung des Aufwandes das Volumen der immateriellen Vermögenswerte ebenfalls ansteigt.

Die zunehmende Abkehr vom Vorsichtsprinzip verlangt verstärkt individuelle Maßnahmen, um die Kapitalerhaltung bei den Kreditnehmern zu verbessern. Befördert wird dies durch den gleichzeitig auftretenden Wandel des deutschen Finanzsystems. Deutschland galt vormals als ein Land, in dem sich Unternehmen schwerpunktmäßig über Hausbanken finanzieren. Als Konsequenz hatten Hausbanken ein stark ausgeprägtes Mitspracherecht in den Unternehmen und waren häufig in Kontrollgremien vertreten; es bestand entsprechend kein großes Interesse an der Verwendung von informati- 


\begin{tabular}{|c|c|c|c|c|}
\hline \multirow[b]{2}{*}{ Kategorie } & \multicolumn{2}{|c|}{ Gläubigerschutzorientierte Rechnungslegung } & \multicolumn{2}{|c|}{ Informatorische Rechnungslegung } \\
\hline & Merkmalsausprägung & $\begin{array}{l}\text { Folgerungen für das } \\
\text { Controlling }\end{array}$ & Merkmalsausprägung & $\begin{array}{l}\text { Folgerungen für das } \\
\text { Controlling }\end{array}$ \\
\hline $\begin{array}{l}\text { Bedeutung von ge- } \\
\text { setzlichen Regulie- } \\
\text { rungsvorschriften für } \\
\text { den Gläubigerschutz }\end{array}$ & Hoch & $\begin{array}{l}\text { Geringe Bedeutung der ex- } \\
\text { ternen Rechnungslegung für } \\
\text { das Controlling }\end{array}$ & Gering & $\begin{array}{l}\text { Hohe Bedeutung der externen } \\
\text { Rechnungslegung für das } \\
\text { Controlling }\end{array}$ \\
\hline $\begin{array}{l}\text { Einfluss der Gläubiger } \\
\text { auf das Bilanzierungs- } \\
\text { verhalten }\end{array}$ & In der Regel nicht vorhanden & $\begin{array}{l}\text { Kaum Schnittmengen zwi- } \\
\text { schen Controlling und exter- } \\
\text { nem Rechnungswesen }\end{array}$ & $\begin{array}{l}\text { Häufig Vorgaben durch } \\
\text { Darlehensgeber }\end{array}$ & $\begin{array}{l}\text { Enge Abstimmung zwischen } \\
\text { Controlling und externem } \\
\text { Rechnungswesen notwendig }\end{array}$ \\
\hline $\begin{array}{l}\text { Maßnahmen zur } \\
\text { Verringerung des In- } \\
\text { solvenzrisikos }\end{array}$ & $\begin{array}{l}\text { Gesetzliche Dividendenaus- } \\
\text { schüttungssperren }\end{array}$ & $\begin{array}{l}\text { Keine Auswirkungen der } \\
\text { Rechnungslegung auf das } \\
\text { Controlling }\end{array}$ & $\begin{array}{l}\text { Einhaltung von finanziel- } \\
\text { len Covenants }\end{array}$ & $\begin{array}{l}\text { Entstehen eines Einkreissys- } \\
\text { tems durch enge Absprache } \\
\text { zwischen Finanzbuchhaltung } \\
\text { und Controlling }\end{array}$ \\
\hline $\begin{array}{l}\text { Kommunikation zwi- } \\
\text { schen Darlehensneh- } \\
\text { mer und Gläubiger }\end{array}$ & $\begin{array}{l}\text { Nicht-standardisierte } \\
\text { Kommunikation }\end{array}$ & Erhöhter Personalaufwand & $\begin{array}{l}\text { Standardisierte Kommu- } \\
\text { nikation }\end{array}$ & Verringerter Personalaufwand \\
\hline Darlehenskosten & $\begin{array}{l}\text { Reduktion der Darlehens- } \\
\text { kosten z. B. durch Vertre- } \\
\text { tung in den Aufsichtsgremien } \\
\text { des Kreditnehmers }\end{array}$ & $\begin{array}{l}\text { Keine Informationsanforde- } \\
\text { rungen an das Controlling }\end{array}$ & $\begin{array}{l}\text { Reduktion der Darle- } \\
\text { henskosten durch Co- } \\
\text { venants }\end{array}$ & $\begin{array}{l}\text { Kostensenkungspotenzial } \\
\text { durch Bereitstellung von Infor- } \\
\text { mationen für Verhandlungen } \\
\text { mit Banken }\end{array}$ \\
\hline $\begin{array}{l}\text { Risiko einer vorzeiti- } \\
\text { gen Darlehenskündi- } \\
\text { gung durch den Gläu- } \\
\text { biger }\end{array}$ & Hohes Maß an Unsicherheit & $\begin{array}{l}\text { Geringe Einwirkungsmög- } \\
\text { lichkeit }\end{array}$ & $\begin{array}{l}\text { Geringes Maß an Unsi- } \\
\text { cherheit }\end{array}$ & $\begin{array}{l}\text { Kostengünstige Kontrolle der } \\
\text { potenziellen Darlehensrisiken } \\
\text { durch standardisierte Repor- } \\
\text { tings }\end{array}$ \\
\hline
\end{tabular}

Abb. 2: Rechnungslegungssystemtypen und Covenants

ven Covenants im Rahmen der Kreditbeziehungen. Mittlerweile jedoch werden in Deutschland die bisherigen engen Beziehungen zwischen Hausbanken und Geschäftskunden vermehrt durch anonymisierte Kreditvertragsbeziehungen ersetzt und die Anteilskonzentration an den Unternehmen sinkt (vgl. Zimmermann et al., 2012). Dies führt ebenfalls zu einem steigenden Bedarf an Covenants, insbesondere informativen Covenants, da Banken nun seltener in den Aufsichts- und Kontrollgremien der Unternehmen vertreten sind und hierdurch einen gestiegenen Informationsbedarf haben.

\section{Implikationen für das Controlling}

Covenants sind für die Unternehmensaktivitäten von hoher Relevanz, da die monetären Konsequenzen aus einer Vertragsverletzung wesentlich sein können und im Extremfall die Existenz des Unternehmens in Frage stellen (vgl. Hannen, 2012). So lassen sich zahlreiche Risiken identifizieren, die durch Verstöße gegen Covenants entstehen können. Eine Auszahlungssperre, Kündigung des Kreditvertrages, Einfrieren von Kreditlinien, Waiver (Verzicht auf das Kündigungs- recht durch Zahlung einer Gebühr), Verschlechterung der Vertragskonditionen, Anpassung der Covenants sowie eine Nachbesicherung seien hier beispielhaft genannt. Welche Maßnahmen Controlling-Abteilungen deutscher Kreditnehmer durch die schrittweise Annäherung der deutschen Bilanzierungsvorschriften an die informatorische Rechnungslegung ergreifen müssen, wird im Folgenden diskutiert. Zunächst werden Merkmalsausprägungen für die beiden Bilanzierungskonzeptionen und die Rolle des Controllings dabei beschrieben (vgl. Abb. 2). Im Anschluss erfolgt eine Diskussion, welche Implikationen sich für deutsche Unternehmen durch den Wandel der deutschen Rechnungslegung weg vom Idealtyp der gläubigerschutzorientierten Rechnungslegung ergeben. Diese Diskussion greift sechs Aspekte auf:

- Bedeutung von gesetzlichen Regulierungsvorschriften für den Gläubigerschutz

- Einfluss der Gläubiger auf das Bilanzierungsverhalten

- Maßnahmen zur Verringerung des Insolvenzrisikos

- Kommunikation zwischen Darlehensnehmer und Gläubiger
Darlehenskosten

- Risiko einer vorzeitigen Darlehenskündigung durch den Gläubiger

Bedeutung von gesetzlichen Regulierungsvorschriften für den Gläubigerschutz

In Ländern, die für eine gläubigerschutzorientierte Bilanzierungskonzeption stehen, ist die Bedeutung der Rechnungslegung für den Gläubigerschutz hoch, da Gesetze und am Vorsichtsprinzip orientierte Rechnungslegungsnormen Gläubigerinteressen sichern. Bei informatorischen Bilanzierungskonzeptionen erfolgt der Gläubigerschutz durch individualvertragliche Vereinbarungen wie Covenants. Wenn sich die Rechnungslegung von einem systemisch organisierten Gläubigerschutz entfernt, steigt die Notwendigkeit, Covenants zu vereinbaren. Dies würde auch eine engere Verknüpfung von Controlling und externem Rechnungswesen erfordern, da Covenants häufig Bilanzierungswahlrechte einschränken und das Controlling die Sicherung der Einhaltung dieser Vorgaben sowie der finanziellen Covenants veranlassen muss. Eine Annäherung des Controllings an das externe Rechnungswesen kann etwa als Wandlung von einem Zweikreissystem hin zu einem Einkreissystem organisiert 


\begin{tabular}{|l|l|}
\hline Einkreissystem & Zweikreissystem \\
\hline $\begin{array}{l}\text { Externes und internes Rechnungswesen eng } \\
\text { miteinander verknüpft }\end{array}$ & $\begin{array}{l}\text { Externes und internes Rechnungswesen } \\
\text { haben kaum Schnittmengen miteinander }\end{array}$ \\
\hline $\begin{array}{l}\text { Bilanz/GuV und Kostenrechnung nahezu identisch, } \\
\text { lediglich geringe Anpassungen notwendig }\end{array}$ & $\begin{array}{l}\text { Zwei getrennte Rechenwerke: } \\
\text { Bilanz/GuV und Kostenrechnung }\end{array}$ \\
\hline $\begin{array}{l}\text { Ressourceneffiziente Aufstellung durch eine } \\
\text { gemeinsame Unternehmensabteilung }\end{array}$ & $\begin{array}{l}\text { Kostenintensiv durch erhöhten } \\
\text { Personal- und hohen IT-Aufwand }\end{array}$ \\
\hline Land mit Einkreissystem: USA & Land mit Zweikreissystem: Deutschland \\
\hline Abb. 3: Einkreissystem und Zweikreissystem im Vergleich
\end{tabular}

werden (vgl. Graves/Berry, 1989; sowie Abb. 3). Diese Entwicklung hin zum Einkreissystem in Deutschland hat neben der verstärkten Covenants-Verwendung weitere Treiber wie die kapitalmarktorientierten Unternehmen selbst, die durch eine einheitliche Finanzsprache organisatorische Vereinfachungen und Kostenreduktionen des Rechnungswesen durchsetzen möchten (vgl. Simons/Weißenberger, 2008). Diese engere Verknüpfung zwischen Controlling und externem Rechnungswesen bedeutet für die Kommunikation innerhalb des Unternehmens eine wesentliche Veränderung: Fand bisher nur eine geringe Abstimmung zwischen den beiden Abteilungen statt, wird es nun notwendig, miteinander häufig zu kommunizieren, um mit den gemeinsamen Schnittmengen (z. B. Einhaltung von Finanzkennzahlen) umgehen zu können.

\section{Einfluss der Gläubiger auf das Bilanzierungsverhalten}

Kreditgeber nehmen in gläubigerschutzorientierten Ländern selten Einfluss auf das Bilanzierungsverhalten. Dies ist nicht erforderlich, da das in den Rechnungslegungsnormen verankerte Vorsichtsprinzip sowie gesetzliche Ausschüttungssperren verhindern, dass Unternehmen zu hohe Gewinne ausweisen bzw. diese Gewinne beispielsweise in Form von Dividenden ausschütten. In informatorischen Rechnungslegungssystemen mit höheren Freiheitsgraden kann die Wirkung von Covenants nur gesichert werden, wenn der Gläubiger auch auf die Bilanzierungspraxis einwirkt. Damit Covenants vergleichbar sind und nicht von den Gläubigern ausgehöhlt werden, indem für sie vorteilhafte Bilanzierungswahlrechte ausgeübt werden, werden Klauseln in Kreditverträge integriert, die die Ausübung von Bilanzierungswahlrechten einschränken und eine Stetigkeit der Bilanzierung verlangen (vgl. Krolak et al., 2009). Auch in Deutschland wird dies zu- nehmend erforderlich, da den bilanzierenden Unternehmen nicht zuletzt durch das BilMoG ein größerer Bilanzierungsspielraum bei der Ertragsrealisation eingeräumt wurde (vgl. Zwirner/Künkele, 2009). Das Controlling muss nun die Einhaltung der Bilanzierungsvorgaben durch das externe Rechnungswesen kontrollieren, da eine Nicht-Einhaltung der Covenants erhebliche finanzielle Konsequenzen nach sich ziehen könnte. Dabei ist eine enge Abstimmung zwischen dem Controlling und dem externem Rechnungswesen unerlässlich.

\section{Maßnahmen zur Verringerung des Insolvenzrisikos}

In Ländern mit staatlich organisierten Elementen des Gläubigerschutzes erfolgt die Kapitalerhaltung durch gesetzlich verankerte Ausschüttungssperren. So dürfen in Deutschland beispielsweise Erträge, die auf die Aktivierung von aktiven latenten Steuern zurückzuführen sind, nicht in Form von Dividenden ausgeschüttet werden $(\$ 274$ Abs. 1 Satz 2 HGB). In Ländern mit einem informatorischen System sichern Covenants die Kapitalerhaltung beispielsweise durch festgesetzte Mindesteigenkapitalquoten oder Rentabilitätskennzahlen. Die Einhaltung der Covenants erfolgt durch das Controlling in Abstimmung mit dem externen Rechnungswesen. Je stärker Covenants künftig in Deutschland Verwendung finden, desto stärker ist dabei die Bedeutung einer Zusammenarbeit zwischen dem Controlling und dem externen Rechnungswesen: So müssen beispielsweise Daten für die Berichtspflichten durch das Controlling aus dem externen Rechnungswesen eingeholt werden. Das Controlling sollte jedoch nicht nur die Berichtspflichten der informativen Covenants erfüllen, sondern ferner ein Frühwarnsystem etablieren, das rechtzeitig auf mögliche Verletzungen von finanziellen Covenants hinweist.

\section{Kommunikation zwischen Darlehens- nehmer und Gläubigern}

In Ländern mit gläubigerschutzorientierten Bilanzierungskonzeptionen erfolgt die Kommunikation zwischen Darlehensnehmer und Gläubiger zumeist nicht-standardisiert. Abweichende Berichtszyklen und Berichtsanforderungen sorgen für eine kostenintensive Kommunikation durch erhöhten Personalaufwand. Standardisierte Prozesse ermöglichen den durch informative Covenants entstehenden Berichtspflichten nachzukommen, indem sie eine ressourceneffiziente und somit günstigere Kommunikation ermöglichen (vgl. Hannen, 2012). Je stärker Covenants in Deutschland zukünftig Einzug in die Kreditvertragsgestaltung erhalten, desto standardisierter sollte die Kommunikation zwischen Gläubigerbanken und Kreditnehmern erfolgen, um Kosten zu reduzieren. Ferner wird die Kommunikation zwischen beiden Parteien intensiviert mit dem Nebeneffekt, dass das Vertrauen zwischen den Vertragsparteien potenziell gestärkt wird (vgl. Theilacker, 2010). Das Controlling des kreditnehmenden Unternehmens hat dabei die Aufgabe, die Daten für die Erfüllung der Berichtspflichten bereitzustellen und die Einhaltung der Vorgaben zu kontrollieren. Dabei ist es unerlässlich, die Vollständigkeit und Korrektheit der durch das Rechnungswesen bereitgestellten Daten zu überprüfen, da diese die Grundlage für die Erstellung der informativen Covenants bilden. Einheitliche und innerhalb des gesamten Konzerns kommunizierte Bilanzierungs-, Bewertungs- und Kontierungsvorschriften verringern die Gefahr von Datenfehlern und sollten durch proaktive und regelmäßige Kontrollen der Daten und Prozesse im Rechnungswesen begleitet werden.

\section{Darlehenskosten}

Informationsasymmetrien werden abgebaut, wenn Hausbanken in Aufsichtsgremien des kreditnehmenden Unternehmens vertreten sind. Dabei haben sie sowohl Zugriff auf alle wesentlichen Finanzinformationen als auch die Möglichkeit zur Kontrolle. Hierdurch verlangen die kreditgebenden Banken geringere Risikoprämien. Diese Beziehung beginnt sich aufzulösen. In solchen Konstellationen ermöglichen Covenants, die Agenturkosten eines Vertragsverhältnisses zu reduzieren. So muss der Kreditnehmer 
Auflagen erfüllen und kann bei NichtEinhaltung der Gebote sanktioniert werden. Dies führt ebenfalls zu sinkenden Risikoprämien, da der Kreditnehmer durch den Gläubiger im Rahmen eines Covenants einen Handlungsrahmen auferlegt bekommt und Informationsasymmetrien verringert werden. Ob es sich jedoch hierbei um eine allokationseffiziente Lösung handelt, hängt von der jeweiligen Gesamtsituation ab. So können durch Kontrollaufwendungen des Gläubigers zusätzliche Kosten entstehen, da dieser die Einhaltung der Covenants überwachen muss. Auch aus Sicht des kreditnehmenden Unternehmens können Covenants zu einer Reduzierung der Kosten beitragen. Durch ein verringertes Insolvenzrisiko kann das Unternehmen bessere Zinskonditionen aushandeln.

Bereits zum Zeitpunkt der Kreditvertragsverhandlungen müssen die Kosten, die durch Covenants entstehen, vom kreditnehmenden Unternehmen berücksichtigt werden, insbesondere wenn mehrere Kreditangebote miteinander verglichen werden sollen: Zum einen entstehen durch die Bereitstellung und Kommunikation von informativen Covenants direkte Kosten, zum anderen bestehen Risiken, die durch Hochrechnungen und Planungsrechungen quantifiziert werden sollten. Dies betrifft insbesondere die Gefahren einer Konditionsanpassung und der Ausübung des Sonderkündigungsrechts durch das Nichteinhalten der finanziellen Covenants.

Werden in Deutschland künftig vermehrt Covenants verwendet, ergibt sich für das Controlling eine neue Situation: Zahlreiche Covenants müssen sodann eingehalten werden, damit keine Sanktionen gegen das Unternehmen verhängt werden. So enthalten Kreditvereinbarungen häufig Regelungen, welche bei Nicht-Einhaltung von finanziellen Covenants erhöhte Zinssätze vorsehen. Damit das kreditnehmende Unternehmen nicht solche zusätzlichen Aufwendungen erleidet, ist die Überwachung der Covenants-Einhaltung durch das Controlling unerlässlich. Grundsätzlich gebietet es sich ferner, regelmäßig eine umfassende Chancen-/Risikoanalyse für bestehende und potenzielle Projekte und Tätigkeiten durchzuführen. Denn es besteht die Gefahr einer verzerrten Risikowahrnehmung: So kann durch eine Fokussierung auf die Einhaltung der Covenants eine bisweilen so risi- koaverse Investitionseinstellung entstehen, dass die Interessen der Eigenkapitalgeber nicht mehr angemessen verfolgt werden.

\section{Risiko einer vorzeitigen Darlehenskündi- gung durch den Gläubiger}

In Ländern mit gläubigerschutzorientierten Bilanzierungskonzeptionen wie Deutschland besteht eine zumindest grundsätzliche Unsicherheit über Darlehenskündigungen bei den Kreditnehmern, da hierfür keine festen Grenzwerte für eine vorzeitige Darlehenskündigung durch den Gläubiger vereinbart sind. Die ungeplante Rückzahlung kann eine Liquiditätslücke verursachen, die möglicherweise bis zu einer Insolvenz führt. Covenants helfen Kreditnehmern bei der Einschätzung, ab wann Gläubiger von einem Kündigungsrecht Gebrauch machen. Insofern reduzieren Covenants die Unsicherheit der Kreditnehmer. Bei einer verstärkten Covenant-Verwendung in Deutschland sollte das Controlling dabei durch standardisierte Reportings kostengünstig auf potenzielle Darlehensrisiken - beispielsweise bei einer kritischen Annäherung an ein finanzielles Covenant wie der Eigenkapitalquote - hinweisen, um eine vorzeitige Kreditvertragskündigung zu verhindern. Sollte eine Nichteinhaltung der Covenants absehbar sein, ist es für das Unternehmen entsprechend ratsam, frühzeitig Gespräche mit den Gläubigerbanken aufzunehmen, da eine Kreditkündigung wesentlich teurer sein dürfte als eine Anpassung der Kreditvertragkonditionen durch die geänderte Risikosituation.

\section{Fazit}

Die unterschiedliche Verbreitung von Covenants kann durch Bilanzierungskonzepte erklärt werden. Während Länder mit informatorischer Rechnungslegung - wenn überhaupt - nur sehr eingeschränkt in standardisierter Form für die Berücksichtigung von Gläubigerinteressen sorgen, werden diese in gläubigerschutzorientierten Ländern durch gesetzliche Regelungen berücksichtigt. Einer individuellen Vereinbarung von Schutzmechanismen in Form von Covenants bedarf es dann nicht. Daher sind Covenants in Ländern mit einem informatorischen Bilanzierungskonzept wie beispielweise den USA verbreiteter als in gläubigerschutzorientierten Ländern wie Deutschland.

Dieses länderspezifische Muster beginnt sich zu verwischen. Die Rechnungslegung in Deutschland befindet sich im Wandel und nähert sich der informatorischen Bilanzierungskonzeption an. Dies, verbunden mit dem Wandel des deutschen Finanzsystems, führt zu einer verstärkten Verwendung von Covenants.

Die zunehmende Verwendung der Covenants in Deutschland hat weitreichende Konsequenzen für deutsche Unternehmen und deren Controlling-Abteilungen. Diese Entwicklung ist aber nicht per se nachteilig für die Unternehmen. Zwar entstehen zusätzliche Kosten durch Informationspflichten, jedoch lassen sich ebenso Vorteile realisieren: eine engere Verzahnung zwischen internem und externem Rechnungswesen und damit verbundene Kosteneinsparungen, eine standardisierte Kommunikation mit den Gläubigerbanken sowie eine verringerte Unsicherheit in Bezug auf die vorzeitige Darlehenskündigung durch den Gläubiger. Um diese Vorteile realisieren zu können, ist ein effizientes und vor allem effektives Controlling unabdingbar, das regelmäßig mit dem externen Rechnungswesen kommuniziert.

Diese Veröffentlichung liefert erste Handlungsvorschläge, wie Unternehmen in Deutschland mit der zunehmenden Verwendung von Covenants umgehen sollten. Es besteht jedoch weiterer Forschungsbedarf: Künftige Forschungsprojekte sollten insbesondere durch qualitative Interviews Best Practices im Controlling für Covenants identifizieren, untersuchen und hieraus Handlungsempfehlungen ableiten.

\section{Keywords: \\ - Accounting Models \\ - Covenants \\ - Covenants-Controlling \\ - Creditor Protection \\ Informative Accounting \\ Summary}

The use of covenants varies across countries, which can be explained by a country's prevalent approach to accounting. Covenants are on the rise in Germany. We discuss reasons for this trend and develop implications for management control systems. 


\section{Literatur}

Bantleon, U./Fengler, P./Peter, A., Einsatz von Covenants im Firmenkundenkreditgeschäft der Volks- und Raiffeisenbanken, in: FinanzBetrieb, 11. Jg. (2009), H. 12, S. 751-759.

Graves, F. O./Berry, M., Accounting's Role in Successful Economic Development: Some Normative Evidence from the German Democratic Republic, in: International Journal of Accounting, 24. Jg. (1989), H. 1, S. 189-220.

Hannen, I., Financial Covenant Management, Controlling und Compliance, in: Der Betrieb, 65. Jg. (2012), H. 40, S. 2233-2240.

Kahle, H., Bilanzieller Gläubigerschutz und internationale Rechnungslegungsstandards, in: Zeitschrift für Betriebswirtschaft, 72. Jg. (2002), H. 7, S. 695-711.

$K P M G$, who is the typical fraudster? KPMG analysis of global patterns of fraud, auf den Seiten der KPMG, http://www.kpmg.com/ $\mathrm{CEE} / \mathrm{en} /$ IssuesAndInsights/ArticlesPublications/Documents/who-is-the-typical-fraudster.pdf, Stand 2011.

Krolak, T./Morzfeld, K./Remmen, J.-D., Financial Covenants als Instrument der Krisenfrüherkennung und der normierten Krisenbewältigung, in: Der Betrieb, 62. Jg. (2009), H. 27, S. 1417-1422.
Meser, M./Veith, S./Zimmermann, J., Disclosure, Enforcement and Capital Market Properties - A Longitudinal Analysis for Germany, in: Schmalenbach Business Review, im Erscheinen 2015.

Omrod, P./Taylor, P., The Impact of the Change to International Accounting Standards on Debt Covenants: A UK Perspective, in: Accounting in Europe, 1. Jg. (2004), H. 1, S. 71-94.

Simons, D./Weißenberger, B. E., Die Konvergenz von externem und internem Rechnungswesen - Kritische Faktoren für die Entwicklung einer partiell integrierten Rechnungslegung aus theoretischer Sicht, in: Betriebswirtschaftliche Forschung und Praxis, 60. Jg. (2008), H.2, S. 137-160.

Smith Jr, C. W./Warner, J. B., On financial contracting: An analysis of bond covenants, in: Journal of Financial Economics, 7. Jg (1979), H. 2, S. 117-161.

Theilacker, B., Covenants - Fluch oder Segen?, in: Zeitschrift für das gesamte Kreditwesen, 63. Jg. (2010), H. 10, S. 503-506.

Velte, P., Reformierung des Kapitalerhaltungssystems auf der Basis von Covenants: Mögliche Konsequenzen für die Ausschüttungsbemessungsfunktion des handelsrechtlichen Jahres- und ,informatorischen Einzelabschlusses" Einzelabschlusses, in: Steuern und Bilanzen, 9. Jg. (2007), H. 17, S. 639 644 .

Zimmermann, J./Werner, J. R./Kilian, J.-P., Covenants in der Kreditvergabe, in: Wirtschaftswissenschaftliches Studium, 41. Jg. (2012), H. 1, S. 3-7.

Zimmermann, J./Werner, J. R./Kilian, J.-P., Wie verbreitet sind Covenants?, in: Die Bank, 51. Jg. (2011), H. 7, S. 20-25.

Zwirner, C./Künkele, K. P., Die Bedeutung der Neuregelungen des BilMoG im Kontext der zunehmenden Anwendung der IFRS: Annäherung statt Übernahme, in: Kapitalmarktorientierte Rechnungslegung, 9. Jg. (2009), H. 11, S. 639-648.

Literaturtipps aus dem Online-Archiv der Controlling:

- Klaus-Peter Franz und Carsten Winkler, IFRS und wertorientiertes Controlling, Ausgabe 8/9/2006, S. 417-423.

- Udo Pyszny, Creditor Relations und Controlling, Ausgabe 7/2007, S. 361366.

- Peter Lorson, Winfried Melcher und Horst Zündorf, Rechnungslegung nach dem Bilanzrechtsmodernisierungsgesetz - Neue Anforderungen für Controller?, Ausgabe 4/5/2010, S. 244-249. 